\title{
Discontinuous Galerkin Immersed Finite Volume Element Method for Anisotropic Flow Models in Porous Medium
}

\author{
Zhong-yan Liu and Huan-zhen Chen \\ School of Mathematical Sciences, Shandong Normal University, Jinan, Shandong 250014, China \\ Correspondence should be addressed to Huan-zhen Chen; chhzh@sdnu.edu.cn
}

Received 24 January 2014; Revised 5 May 2014; Accepted 14 May 2014; Published 1 June 2014

Academic Editor: Bashir Ahmad

Copyright (C) 2014 Z.-y. Liu and H.-z. Chen. This is an open access article distributed under the Creative Commons Attribution License, which permits unrestricted use, distribution, and reproduction in any medium, provided the original work is properly cited.

By choosing the trial function space to the immersed finite element space and the test function space to be piecewise constant function space, we develop a discontinuous Galerkin immersed finite volume element method to solve numerically a kind of anisotropic diffusion models governed by the elliptic interface problems with discontinuous tensor-conductivity. The existence and uniqueness of the discrete scheme are proved, and an optimal-order energy-norm estimate and $L^{2}$-norm estimate for the numerical solution are derived.

\section{Introduction}

Let us consider the following elliptic interface problems in a convex domain $\Omega \subset R^{2}$ :

$$
\begin{gathered}
-\nabla \cdot(\mathbb{B} \nabla u)=f, \quad(x, y) \in \Omega, \\
u=0, \quad(x, y) \in \partial \Omega,
\end{gathered}
$$

where $\Omega$ is separated into two subdomains $\Omega^{+}$and $\Omega^{-}$by interface $\Gamma \in C^{2}$, see Figure 1 for an illustration, and $f \in$ $L^{2}(\Omega) ; u$ satisfies the following homogenous jump conditions on the interface $\Gamma$ :

$$
[u],\left[\mathbb{B} \frac{\partial u}{\partial \mathbf{n}}\right]=0 .
$$

Equation (1) describes many real diffusion processes in fluid dynamics and engineering applications, such as the miscible displacement with discontinues conductivity due to complex strata or multiphase flux. It is significant to seek efficiently the numerical solution to the interface problems for better understanding of the mechanism of the flow process and conducting engineering practice.

When $\mathbb{B}(x)$ is a scale function, which corresponds to an isotropic flow case, two classes of numerical methods were developed to approximate (1) in terms of the meshes. One is the fitted finite element or fitted difference method [13], which restricts the mesh to be aligned with the smooth interface $\Gamma$. Consequently, the fitted methods are costly for more complicated time dependent problems in which the interface moves with time and repeated grid generation is called for. The other one is the immersed interface difference or finite element methods in which the Cartesian grid is naturally used even though it cannot match a curved interface. Although the immersed difference methods $[4,5]$ were demonstrated to be very effective, convergence analysis of related finite difference methods is extremely difficult and is still open. On the other hand, the immersed finite element method (IFE) was developed, which allows the interface to go through the interior of the element; see the references [6-9] and the references therein. Numerical experiments demonstrated an optimal order of the errors. Once again, it is not easy to analyze this method. Further, to preserve the conservative characteristics of the interface model (1), [10] developed an immersed finite volume element (IFVE) method by combining the finite volume element method [11$16]$ and the immersed finite element method.

In realistic diffusion processes, the interface problem (1) displays much often its anisotropic type. That is, the conductivity $\mathbb{B}(x)$ becomes a tenser-formed function. The goals of this paper are as follows: (1) to develop a discontinuous Galerkin-immersed interface-finite volume element 


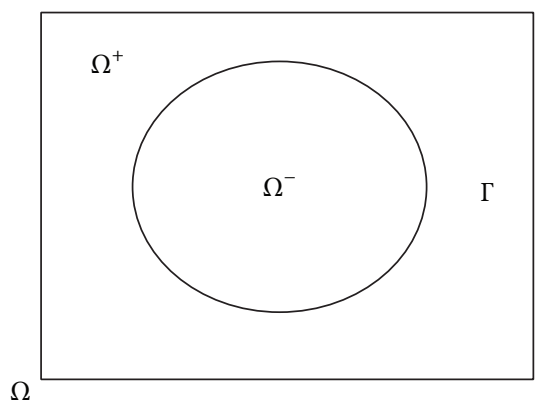

FIGURE 1

(DGIFVE) method for the second-order elliptic problems with tensor-formed conductivity $\mathbb{B}(\mathbf{x})$ defined by

$$
\mathbb{B}(\mathbf{x})=\mathbb{B}^{l}(\mathbf{x})=\left(\begin{array}{cc}
m^{l} & s^{l} \\
s^{l} & n^{l}
\end{array}\right),
$$

where $\mathbf{x} \in \Omega^{l}, m^{l}>0, n^{l}>0, m^{l} n^{l}>\left(s^{l}\right)^{2}, l=+,-$; by doing so, we can use the ability of the penalty term in discontinuous Galerkin method to control the integrals on an element boundary, in order to prove the solvability of the scheme and derive easily an optimal-order error analysis, and we can use the conservation characteristics of the finite volume element method to construct a conservationpreserved numerical method; (2) to prove the existence and uniqueness of the proposed discontinuous Galerkinimmersed interface-finite volume element procedure based on the nonconforming interface finite element space for anisotropic flow model [17]; (3) to establish its optimal-order energy-norm estimate and $L^{2}$-norm estimate.

This paper is organized as follows. In the next section, we will introduce the trial function space and its approximation properties on primal triangulation. In Section 3, we will formulate the DGIFVE procedure. In Section 4, we will introduce some important lemmas. In Section 5, we will prove the existence and uniqueness of the solution of the discrete scheme. In Section 6, we will derive the convergence analysis.

Throughout this paper, the symbol $C$ will be used as a generic positive constant independent of $h$ and may have different values at different places.

\section{The Construction of the Trial Function Space}

In this section, we recall the definitions of IFE spaces discussed in [7]. Let $\mathscr{T}_{h}=\{K\}$ be a regular triangulation of $\Omega$ with the diameter size $h$. We can separate the triangles on a partition into two classes:

(1) interface element: the interface $\Gamma$ passes through the interior of $K$;

(2) noninterface element: the interface does not intersect with this triangle, or it intersects with this triangle but does not separate its interior into two nontrivial subsets. Let $\mathscr{T}_{h}^{n}$ be the collection of all noninterface elements and let $\mathscr{T}_{h}^{m}$ be the collection of all interface elements. We will use $A_{i}=\left(x_{i}, y_{i}\right), i=1,2,3$ to denote the vertices of $T$, and we will use $\overline{D E}$ to denote the line segment connecting the intersection of the interface and the edges of a triangle $K$. This line segment $\overline{D E}$ divides $T$ into two parts $K^{+}$and $K^{-}$with $K=K^{+} \cup K^{-} \cup \overline{D E}$ (see Figure 2).

For the analysis, we introduce the spaces

$$
\begin{gathered}
\widetilde{H}^{2}(\Omega)=\left\{u \in H_{0}^{1}(\Omega): u \in H^{2}\left(\Omega^{s}\right), s=+,-\right\}, \\
\widetilde{H}^{2}(K)=\left\{u \in H^{1}(K): u \in H^{2}\left(K \cap \Omega^{s}\right), s=+,-\right\}
\end{gathered}
$$

equipped with the norms

$$
\begin{gathered}
\|u\|_{\widetilde{H}^{2}(\Omega)}^{2}=\|u\|_{H^{2}\left(\Omega^{+}\right)}^{2}+\|u\|_{H^{2}\left(\Omega^{-}\right)}^{2}, \\
\|u\|_{\widetilde{H}^{2}(K)}^{2}=\|u\|_{H^{2}\left(K \cap \Omega^{+}\right)}^{2}+\|u\|_{H^{2}\left(K \cap \Omega^{-}\right)}^{2},
\end{gathered}
$$

where $H^{m}=W_{2}^{m}(m=1,2)$ is the standard Sobolev spaces. In order to define the bilinear formulation, we introduce the broken Sobolev space $H^{1}\left(\mathscr{T}_{h}\right)=\left\{u \in L^{2}(\Omega): \forall K \in\right.$ $\mathscr{T}_{h},\left.u\right|_{K} \in H^{1}(K)$ and $\left.\forall e c \partial K \cap \partial \Omega \neq \emptyset,\left.u\right|_{e}=0\right\}$.

For a noninterface element $K$, we use the standard linear shape functions on $K$ whose degrees of freedom are functional values on the vertices of $K$, and we use $\bar{S}_{h}(K)$ to denote the linear spaces spanned by the three nodal basis functions on $K$ as follows:

$$
\bar{S}_{h}(K)=\operatorname{span}\left\{\phi_{i} \in P_{1}(K): i=1,2,3\right\},
$$

where $P_{1}(K)$ is the linear space on $K$. For this space, we have the following estimate of the interpolant:

$$
\left\|u-\Pi_{h} u\right\|_{L^{2}(K)}+h\left\|u-\Pi_{h} u\right\|_{H^{1}(K)} \leqslant C h^{2}\|u\|_{H^{2}(K)},
$$

where $\Pi_{h}: H^{2}(K) \rightarrow \bar{S}_{h}(K)$ is the interpolation operator. We use $\bar{S}_{h}(\Omega)$ to denote the space of the conforming piecewise linear polynomials on the domain $\mathscr{T}_{h}^{n}$.

For an interface element $K$ whose geometric configuration is given in Figure 3 in which $A_{1}=(0,0)^{T}, A_{2}=$ $\left(h_{1}, 0\right)^{T}, A_{3}=\left(0, h_{2}\right)^{T}$, the interface points $D=\left(b h_{1}, 0\right)^{T}$ and $E=\left(0, a h_{2}\right)^{T}$, where $0<a \leqslant 1$ and $0<b \leqslant 1$. Let $\phi_{i}, i=1,2,3$, denote the usual Lagrange nodal basis function associated with the vertex $A_{i}, i=1,2,3$, respectively. Here we assume that the ratio $r=h_{1} / h_{2}$ is bounded below and above by some constants.

By $\widehat{\phi}\left(A_{i}\right)=V_{i}, i=1,2,3$, we can construct the basis function $\widehat{\phi}(\mathbf{x})$ on an interface element $K$ as follows:

$$
\widehat{\phi}(\mathbf{x})= \begin{cases}\hat{\phi}^{-}(\mathbf{x})=V_{1} \phi_{1}+C_{1} \phi_{2}+C_{2} \phi_{2}, & \mathbf{x}=(x, y) \in K^{-}, \\ \hat{\phi}^{+}(\mathbf{x})=C_{3} \phi_{1}+V_{2} \phi_{2}+V_{3} \phi_{2}, & \mathbf{x}=(x, y) \in K^{+} .\end{cases}
$$

Satisfy

$$
\begin{gathered}
\widehat{\phi}^{+}(D)=\widehat{\phi}^{-}(D), \quad \widehat{\phi}^{+}(E)=\widehat{\phi}^{-}(E), \\
\mathbb{B}^{+} \frac{\partial \hat{\phi}^{+}}{\partial \mathbf{n}_{\overline{D E}}}=\mathbb{B}^{-} \frac{\partial \widehat{\phi}^{-}}{\partial \mathbf{n}_{\overline{D E}}},
\end{gathered}
$$

where $\mathbf{n}_{\overline{D E}}$ is the unit normal vector on the linesegment $\overline{D E}$. 


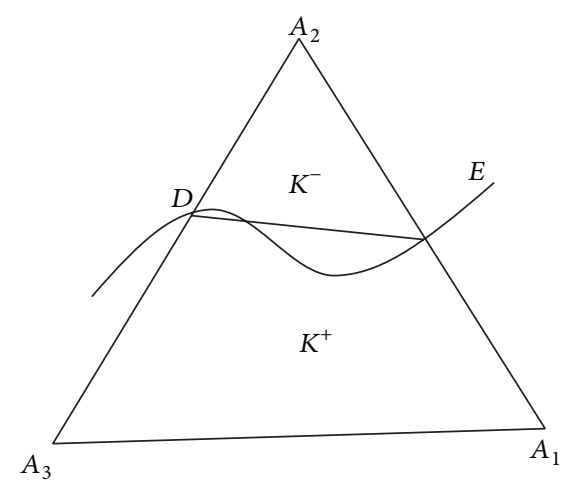

FIGURE 2

By $[17,18]$, we have the following conclusions.

Lemma 1. When $s^{l} \geqslant 0, l=+,-$, the piecewise linear function $\widehat{\phi}(x, y)$ defined by (8) is uniquely decided by three conditions in (9).

Remark 2. By [17], the condition $s^{l} \geq 0, l=+,-$ is necessary in Lemma 1. For some specially selected entries of $\mathbb{B}^{l}$ and the intersection points of the interface with the edges on $K, \widehat{\phi}(\mathbf{x})$ satisfying (9) is uniquely undetermined by $\widehat{\phi}\left(A_{i}\right)=V_{i}, i=$ $1,2,3$.

Based on the above results, the finite element space $\widehat{S}_{h}(K)$ on a typical interface element $K \in \mathscr{T}_{h}^{m}$ is defined by

$$
\widehat{S}_{h}(K)=\{\widehat{\phi}: \widehat{\phi} \text { is piecewise linear and satisfies (9) }\} \text {. }
$$

We call $\widehat{S}_{h}(K)$ the immersed interface element space. For any $u \in \widetilde{H}^{2}(\Omega)$ and $K \in \mathscr{T}_{h}^{m}$, we define $\left.\left(\Pi_{h} u\right)\right|_{K}=\Pi_{h}\left(\left.u\right|_{K}\right) \epsilon$ $\widehat{S}_{h}(K)$ by

$$
\Pi_{h} u\left(A_{i}\right)=u\left(A_{i}\right), \quad i=1,2,3,
$$

and we call $\Pi_{h} u$ the interpolant of $u$ in $\widehat{S}_{h}(K)$. Similar to [7], we have an estimate of the interpolant given in the following theorem.

Theorem 3. For $\forall K \in \mathscr{T}_{h}^{m}$, there exists a constant $C>0$ such that the interpolation operator $\Pi_{h}: \widetilde{H}^{2}(K) \rightarrow \widehat{S}_{h}(K)$ satisfies

$$
\begin{array}{r}
\left\|u-\Pi_{h} u\right\|_{L^{2}(K)}+h\left\|u-\Pi_{h} u\right\|_{H^{1}(K)} \leqslant C h^{2}\|u\|_{\widetilde{H}^{2}(K)}, \\
\forall u \in \widetilde{H}^{2}(K) .
\end{array}
$$

Finally, we define trial function space $S_{h}(\Omega)$ as the collection of functions such that

$$
\begin{gathered}
\left.\phi\right|_{K} \in \bar{S}_{h}(K), \quad K \text { is a noninterface element, } \\
\left.\phi\right|_{K} \in \widehat{S}_{h}(K), \quad K \text { is an interface element. }
\end{gathered}
$$

The space $S_{h}(\Omega) \subset L^{2}(\Omega)$ is a subspace of $H^{1}\left(\mathscr{T}_{h}\right)$. We also use the space $S_{0 h}(\Omega)=\left\{v_{h} \in S_{h}(\Omega),\left.v_{h}\right|_{\partial \Omega}=0\right\}$.

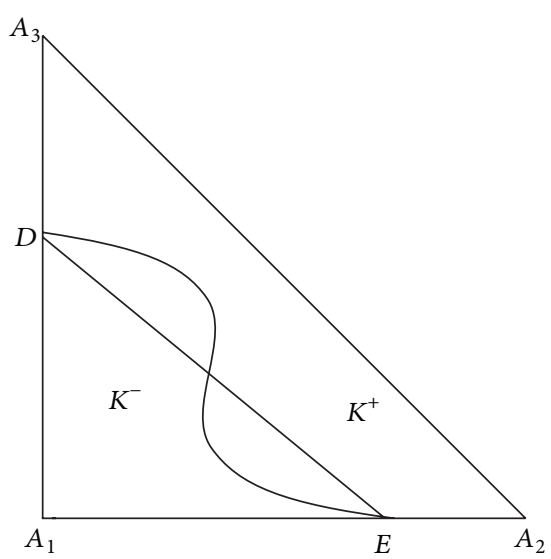

Figure 3

\section{DGIFVE Procedure}

In this section, we will construct a dual grid $\mathscr{T}_{h}^{*}$ based on $\mathscr{T}_{h}$. Assume that the triangulation $\mathscr{T}_{h}$ is quasi-uniform. For a given triangle $K \in \mathscr{T}_{h}$, we divide $K \in \mathscr{T}_{h}$ into three triangles by connecting the barycenter $Q$ and the three corners of the triangle as shown in Figure 4 . Let $\mathscr{T}_{h}^{*}$ consist of all these triangles $T$.

For the $\mathscr{T}_{h}^{*}$, we define the test function space as follows:

$$
S_{h}^{*}(\Omega)=\left\{\phi(\mathbf{x})|\phi(\mathbf{x})|_{T}=\text { constant, } \forall T \in \mathscr{T}_{h}^{*}\right\} .
$$

Analogous to the operator $\Pi_{h}$, we introduce the interpolation operator $\gamma_{h}: H_{h}=S_{h}(\Omega)+\widetilde{H}^{2}(\Omega) \cap H_{0}^{1}(\Omega) \longrightarrow S_{h}^{*}(\Omega)$ defined by, for $\forall v \in H_{h}$,

$$
\left.\gamma_{h} v\right|_{T}=\frac{1}{|e|} \int_{e} v(\mathbf{x}) d \mathbf{x}, \quad \mathbf{x} \in T
$$

Let $e$ be an interior edge shared by two elements $K_{1}$ and $K_{2}$ in $\mathscr{T}_{h}$. Define the unite normal vectors $\mathbf{n}_{1}$ and $\mathbf{n}_{2}$ on $e$ pointing exterior to $K_{1}$ and $K_{2}$, respectively. For scalar $v$ function and vector function $\mathbf{q}$, we define their average $\{\cdot\}$ and jump [.] on $e$, as follows (see [19]):

$$
\begin{aligned}
\left.\{v\}\right|_{e}=\frac{1}{2}\left(\left.v\right|_{\partial K_{1}}+\left.v\right|_{\partial K_{2}}\right), & {\left.[v]\right|_{e}=\left.v\right|_{\partial K_{1}} \cdot \mathbf{n}_{1}+\left.v\right|_{\partial K_{2}} \cdot \mathbf{n}_{2}, } \\
\left.\{\mathbf{q}\}\right|_{e}=\frac{1}{2}\left(\left.\mathbf{q}\right|_{\partial K_{1}}+\left.\mathbf{q}\right|_{\partial K_{2}}\right), & {\left.[\mathbf{q}]\right|_{e}=\left.\mathbf{q}\right|_{\partial K_{1}} \cdot \mathbf{n}_{1}+\left.\mathbf{q}\right|_{\partial K_{2}} \cdot \mathbf{n}_{2} . }
\end{aligned}
$$

If $e$ is an edge on the boundary of $\Omega$, we define

$$
\left.\{v\}\right|_{e}=v,\left.\quad[\mathbf{q}]\right|_{e}=\mathbf{q} \cdot \mathbf{n} .
$$

Let $\varepsilon_{h}$ denote the union of the boundaries of the triangle $K$ of $\mathscr{T}_{h}$ and let $\varepsilon_{h}^{0}:=\varepsilon_{h} \backslash \partial \Omega, \varepsilon_{h}^{*}$ be the union of the boundaries cutting by the $\Gamma$. A straightforward computation gives

$$
\sum_{K \in \mathscr{T}_{h}} \int_{\partial K} v \mathbf{q} \cdot \mathbf{n} d s=\sum_{e \in \varepsilon_{h}} \int_{e}[v]\{\mathbf{q}\} d s+\sum_{e \in \varepsilon_{h}^{0}} \int_{e}\{v\}[\mathbf{q}] d s .
$$




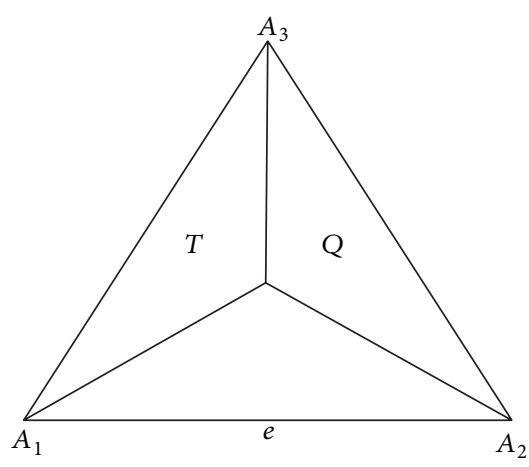

(a)

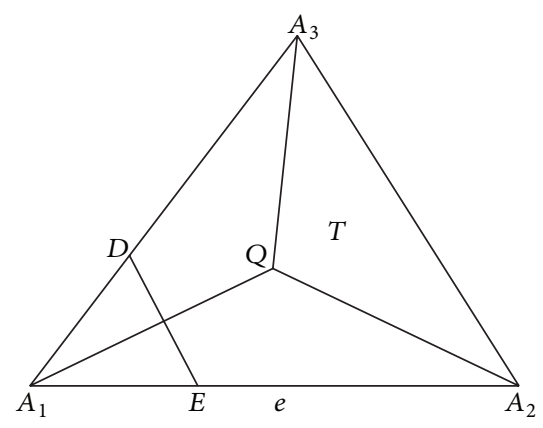

(b)

FIgURE 4: (a) $K$ is a noninterface element and (b) $K$ is an interface element.

We multiply (1) by $v_{h} \in S_{h}^{*}(\Omega)$; using $\left.[\mathbb{B} \nabla u]\right|_{\Gamma}=0$ and Green's formula, we have

$$
-\sum_{T \in \mathscr{T}_{h}^{*}} \int_{\partial T} \mathbb{B} \nabla u \cdot \mathbf{n} v_{h} d s=\left(f, v_{h}\right),
$$

where $\mathbf{n}$ is the unit outward normal vector on $\partial T$. Let $T_{j} \in$ $\mathscr{T}_{h}^{*}(j=1,2,3)$ be three triangles in $K \in \mathscr{T}_{h}$. Then, we have

$$
\begin{aligned}
& \sum_{T \in \mathscr{T}_{h}^{*}} \int_{\partial T} \mathbb{B} \nabla u \cdot \mathbf{n} v_{h} d s \\
& \quad=\sum_{K \in \mathscr{T}_{h}} \sum_{j=1} \int_{\partial T_{j}} \mathbb{B} \nabla u \cdot \mathbf{n} v_{h} d s \\
& \quad=\sum_{K \in \mathscr{T}_{h}} \sum_{j=1} \int_{A_{j+1} \mathrm{QA}} \mathbb{B} \nabla u \cdot \mathbf{n} v_{h} d s+\sum_{K \in \mathscr{T}_{h}} \int_{\partial K} \mathbb{B} \nabla u \cdot \mathbf{n} v_{h} d s .
\end{aligned}
$$

Using (18) and the fact that $[\mathbb{B} \nabla u]=0,(20)$ becomes

$$
\begin{aligned}
& \sum_{T \in \mathscr{T}_{h}^{*}} \int_{\partial T} \mathbb{B} \nabla u \cdot \mathbf{n} v_{h} d s \\
& \quad=\sum_{K \in \mathscr{T}_{h}} \sum_{j=1} \int_{A_{j+1} Q A_{j}} \mathbb{B} \nabla u \cdot \mathbf{n} v_{h} d s+\sum_{e \in \varepsilon_{h}} \int_{e}\{\mathbb{B} \nabla u\}\left[v_{h}\right] d s .
\end{aligned}
$$

By (19) and (21), we can get

$$
\begin{aligned}
-\sum_{K \in \mathscr{T}_{h}} & \sum_{j=1} \int_{A_{j+1} \mathrm{QA}} \mathbb{B} \nabla u \cdot \mathbf{n} v_{h} d s \\
& -\sum_{e \in \varepsilon_{h}} \int_{e}\{\mathbb{B} \nabla u\}\left[v_{h}\right] d s=\left(f, v_{h}\right) .
\end{aligned}
$$

By the definition of $\gamma_{h}$, the discontinuous Galerkin immersed finite volume element formulation is equivalent to finding $u_{h} \in S_{0 h}(\Omega)$ such that

$$
a_{h}^{*}\left(u_{h}, \gamma_{h} \omega_{h}\right)=\left(f, \gamma_{h} \omega_{h}\right), \quad \omega_{h} \in S_{0 h}(\Omega),
$$

where

$$
\begin{aligned}
& a_{h}^{*}\left(u_{h}, \gamma_{h} \omega_{h}\right) \\
& =-\sum_{K \in \mathscr{T}_{h}} \sum_{j=1} \int_{A_{j+1} \mathrm{QA}} \mathbb{B} \nabla u_{h} \cdot \mathbf{n} \gamma_{h} \omega_{h} d s \\
& \quad-\sum_{e \in \varepsilon_{h}} \int_{e}\left\{\mathbb{B} \nabla u_{h}\right\}\left[\gamma_{h} \omega_{h}\right] d s \\
& \quad+\sum_{e \in \varepsilon_{h}} \frac{\sigma_{0}}{h_{e}} \int_{e}\left[u_{h}\right]\left[\omega_{h}\right] d s
\end{aligned}
$$

is the bilinear formulation defined on $S_{0 h}(\Omega) \times S_{0 h}(\Omega)$, and in addition to penalty term $\sum_{e \in \varepsilon_{h}}\left(\sigma_{0} / h_{e}\right) \int_{e}\left[u_{h}\right]\left[\omega_{h}\right] d s$, the penalty parameter $\sigma_{0}>0$. Since $\left[\gamma_{h} u\right]_{e}=0$, it is easy to see that $u$ satisfies the solution of (1) as follows:

$$
a_{h}^{*}\left(u, \gamma_{h} \omega_{h}\right)=f\left(u, \gamma_{h} \omega_{h}\right) .
$$

Let

$$
A\left(u_{h}, \omega_{h}\right)=-\sum_{K \in \mathscr{T}_{h}} \sum_{j=1}^{3} \int_{A_{j+1} Q A_{j}} \mathbb{B} \nabla u_{h} \cdot \mathbf{n} \gamma_{h} \omega_{h} d s .
$$

If $K \in \mathscr{T}_{h}^{n}$, we have by $\nabla \cdot\left(\mathbb{B} \nabla u_{h}\right)=0, u_{h} \in S_{0 h}(\Omega)$

$$
\begin{aligned}
0= & \int_{K} \nabla \cdot\left(\mathbb{B} \nabla u_{h}\right) \gamma_{h} \omega_{h} d x \\
= & \sum_{j=1}^{3} \int_{A_{j+1} Q A_{j}} \mathbb{B} \nabla u_{h} \cdot \mathbf{n} \gamma_{h} \omega_{h} d s \\
& +\sum_{j=1}^{3} \int_{A_{j} A_{j+1}} \mathbb{B} \nabla u_{h} \cdot \mathbf{n} \gamma_{h} \omega_{h} d s .
\end{aligned}
$$

Then,

$$
-\sum_{j=1}^{3} \int_{A_{j+1} \mathrm{Q} A_{j}} \mathbb{B} \nabla u_{h} \cdot \mathbf{n} \gamma_{h} \omega_{h} d s=\sum_{j=1}^{3} \int_{A_{j} A_{j+1}} \mathbb{B} \nabla u_{h} \cdot \mathbf{n} \gamma_{h} \omega_{h} d s .
$$


Similarly,

$$
\begin{aligned}
0 & =-\int_{K} \nabla \cdot\left(\mathbb{B} \nabla u_{h}\right) \omega_{h} d x \\
& =-\sum_{j=1}^{3} \int_{A_{j} A_{j+1}} \mathbb{B} \nabla u_{h} \cdot \mathbf{n} \omega_{h} d s+\int_{K} \mathbb{B} \nabla u_{h} \cdot \nabla \omega_{h} d x .
\end{aligned}
$$

We find that

$$
\int_{\partial K} \mathbb{B} \nabla u_{h} \cdot \mathbf{n}\left(\omega_{h}-\gamma_{h} \omega_{h}\right) d s=0,
$$

due to the fact that $\mathbb{B} \nabla u_{h}$ is a constant vector on each edge and the definition of $\gamma_{h}$. Thus, we can get

$$
\int_{K} \mathbb{B} \nabla u_{h} \nabla \omega_{h} d x=-\sum_{j=1}^{3} \int_{A_{j+1} Q A_{j}} \mathbb{B} \nabla u_{h} \cdot \mathbf{n} \gamma_{h} \omega_{h} d s
$$

following from (28) and (29). For $K \in \mathscr{T}_{h}^{m}$ (see Figure 4(b)), it follows from the same arguments above and the $\left.\left[B \nabla u_{h}\right]\right|_{\overline{D E}}=$ 0 that

$$
\begin{aligned}
\int_{K} \mathbb{B} \nabla u_{h} \nabla \omega_{h} d x=- & \sum_{j=1}^{3} \int_{A_{j+1} Q A_{j}} \mathbb{B} \nabla u_{h} \cdot \mathbf{n} \gamma_{h} \omega_{h} d s \\
& +\left\{\int_{\overline{A_{1} D}} \mathbb{B}^{+} \nabla u_{h}^{+} \cdot \mathbf{n}\left(\gamma_{h} \omega_{h}-\omega_{h}^{+}\right) d s\right. \\
& +\int_{\overline{D A_{2}}} \mathbb{B}^{-} \nabla u_{h}^{-} \cdot \mathbf{n}\left(\gamma_{h} \omega_{h}-\omega_{h}^{-}\right) d s \\
& +\int_{\overline{A_{2} E}} \mathbb{B}^{-} \nabla u_{h}^{-} \cdot \mathbf{n}\left(\gamma_{h} \omega_{h}-\omega_{h}^{-}\right) d s \\
& \left.+\int_{\overline{E A_{3}}} \mathbb{B}^{+} \nabla u_{h}^{+} \cdot \mathbf{n}\left(\gamma_{h} \omega_{h}-\omega_{h}^{+}\right) d s\right\} .
\end{aligned}
$$

Summarizing the results above, we have

$$
\begin{aligned}
A\left(u_{h}, \omega_{h}\right)= & -\sum_{K} \sum_{j=1}^{3} \int_{A_{j+1} \mathrm{QA} A_{j}} \mathbb{B} \nabla u_{h} \cdot \mathbf{n} \gamma_{h} \omega_{h} d s \\
= & \sum_{K \in \mathscr{T}_{h}} \int_{K} \mathbb{B} \nabla u_{h} \nabla \omega_{h} d x \\
& -\sum_{K \in \mathscr{T}_{h}^{m}} \sum_{e \in \varepsilon_{h}^{*}} \int_{e} \mathbb{B} \nabla u_{h} \cdot \mathbf{n}\left(\omega_{h}-\gamma_{h} \omega_{h}\right) d s \\
= & \sum_{K \in \mathscr{T}_{h}} \int_{K} \mathbb{B} \nabla u_{h} \nabla \omega_{h} d x \\
& -\sum_{e \in \varepsilon_{h}^{*}} \int_{e}\left[\mathbb{B} \nabla u_{h}\right]\left\{\omega_{h}-\gamma_{h} \omega_{h}\right\} d s \\
& -\sum_{e \in \varepsilon_{h}^{*}} \int_{e}\left\{\mathbb{B} \nabla u_{h}\right\}\left[\omega_{h}-\gamma_{h} \omega_{h}\right] d s .
\end{aligned}
$$

Thus, (24) can be written by

$$
\begin{aligned}
a_{h}^{*}\left(u_{h}, \gamma_{h} \omega_{h}\right)= & A\left(u_{h}, \omega_{h}\right)-\sum_{e \in \varepsilon_{h}} \int_{e}\left\{\mathbb{B} \nabla u_{h}\right\}\left[\gamma_{h} \omega_{h}\right] d s \\
& +\sum_{e \in \varepsilon_{h}} \frac{\sigma_{0}}{|e|^{\alpha_{0}}} \int_{e}\left[u_{h}\right]\left[\omega_{h}\right] d s .
\end{aligned}
$$

\section{Some Lemmas}

We define a norm $\||\cdot|||$ for $H_{h}$ as follows:

$$
\||v|\|^{2}=|v|_{1, h}^{2}+\sum_{e} \frac{1}{h_{e}} \int_{e}[v]^{2} d s+\sum_{e \in \varepsilon_{h}^{*}} h_{e} \int_{e}[\beta \nabla v]^{2} d s .
$$

In order to prove the existence and uniqueness of the solution to (24) and conduct its convergence analysis, we need the following lemmas.

Lemma 4. The operator $\gamma_{h}$ in (15) has the following properties:

$$
\begin{gathered}
\int_{e}\left(\omega-\gamma_{h} \omega\right) d s=0, \quad \forall \omega \in H_{h}, \quad \forall e \in \varepsilon_{h} ; \\
{\left.[\omega]\right|_{e}=\left.0 \Longrightarrow\left[\gamma_{h} \omega\right]\right|_{e}=0, \quad \forall \omega \in H_{h} ;} \\
\left\|\gamma_{h} \omega-\omega\right\|_{0, K} \leqslant C h_{K}|\omega|_{1, K}, \quad \forall K \in \mathscr{T}_{h}, \omega \in H_{h} .
\end{gathered}
$$

Proof. Obviously, (36) and (37) follow from the definition of $\gamma$ in (15). We only prove (38) below.

Let $K$ be a noninterface element; we have the conclusion (38) by [12]. Therefore, we focus (38) on interface element $K$ (Figure 4(b)). For $\forall \omega \in S_{h}(\Omega)$, we have the following form:

$$
\omega= \begin{cases}\omega^{+}=a_{0}+b_{0} x+c_{0} y, & \mathbf{x}=(x, y) \in K^{+}, \\ \omega^{-}=a_{1}+b_{1} x+c_{1} y, & \mathbf{x}=(x, y) \in K^{-} .\end{cases}
$$

The jump conditions on $\overline{D E}$ lead to (see [7])

$$
\nabla \omega^{+}=\left(\begin{array}{cc}
\mathbf{n}_{y}^{2}+\rho \mathbf{n}_{x}^{2} & (\rho-1) \mathbf{n}_{x} \mathbf{n}_{y} \\
(\rho-1) \mathbf{n}_{x} \mathbf{n}_{y} & \mathbf{n}_{x}^{2}+\rho \mathbf{n}_{y}^{2}
\end{array}\right) \nabla \omega^{-},
$$

or

$$
\nabla \omega^{+}=N_{\overline{D E}}^{-} \nabla \omega^{-},
$$

where $\mathbf{n}_{\overline{D E}}=\left(\mathbf{n}_{x}, \mathbf{n}_{y}\right)^{T}$ and $\rho=\left(\beta^{-} / \beta^{+}\right)$. We know that

$$
\left\|\omega-\gamma_{h} \omega\right\|_{0, K}^{2}=\sum_{j=1}^{3} \int_{T_{j}}\left(\omega-\gamma_{h} \omega\right)^{2} d \mathbf{x},
$$

where

$$
\begin{aligned}
\int_{T_{i}} & \left(\omega-\gamma_{h} \omega\right)^{2} d \mathbf{x} \\
= & \int_{T_{i}^{+}}\left(\omega^{+}-\gamma_{h} \omega\right)^{2} d \mathbf{x}+\int_{T_{i}^{-}}\left(\omega^{-}-\gamma_{h} \omega\right)^{2} d \mathbf{x}, \quad i=1,2 .
\end{aligned}
$$


Since $\omega$ is continuous on $\overline{A_{1} A_{2}}$, there exists a point $\xi$ such that

$$
\gamma_{h} \omega \mid \overline{A_{1} A_{2}}=\omega(\xi) .
$$

We suppose that $\xi$ fall on $\overline{A_{1} D}$; then, we have

$$
\begin{aligned}
\int_{T_{1}} & \left(\omega-\gamma_{h} \omega\right)^{2} d \mathbf{x} \\
= & \int_{T_{1}^{+}}\left(\omega^{+}-\omega^{+}(\xi)\right)^{2} d \mathbf{x}+\int_{T_{1}^{-}}\left(\omega^{-}-\omega^{+}(\xi)\right)^{2} d \mathbf{x} \\
= & \int_{T_{1}^{+}}\left(\omega^{+}-\omega^{+}(\xi)\right)^{2} d \mathbf{x} \\
& +\int_{T_{1}^{-}}\left(\omega^{-}-\omega^{-}(D)+\omega_{h}^{+}(D)-\omega^{+}(\xi)\right)^{2} d \mathbf{x},
\end{aligned}
$$

where we used $\omega^{-}(D)=\omega^{+}(D)$. Because $\omega^{+}(\mathbf{x})$ and $\omega^{-}(\mathbf{x})$ are linear polynomial, we have

$$
\begin{gathered}
\omega^{+}(\mathbf{x})=\omega^{+}(\xi)+\nabla \omega^{+}(\mathbf{x}-\xi), \\
\omega^{-}(\mathbf{x})=\omega^{-}(D)+\nabla \omega^{-}(\mathbf{x}-D) .
\end{gathered}
$$

From these expansions of $\omega$ and (41), we have

$$
\begin{aligned}
\int_{T_{1}} & \left(\omega-\gamma_{h} \omega\right)^{2} d \mathbf{x} \\
= & \int_{T_{1}^{+}}\left(\nabla \omega^{+}(\mathbf{x}-\xi)\right)^{2} d \mathbf{x} \\
& +\int_{T_{1}^{-}}\left(\nabla \omega^{-}(\mathbf{x}-D)+N_{\overline{D E}}^{-} \nabla \omega^{-}(D-\xi)\right)^{2} d \mathbf{x} .
\end{aligned}
$$

Then,

$$
\int_{T_{1}}\left(\omega-\gamma_{h} \omega\right)^{2} d \mathbf{x} \leqslant C h^{2}|\omega|_{1, T_{1}}^{2}
$$

If $\xi$ is on $\overrightarrow{D A}_{2}$, similarly, we also have (48). Analogously, we can have the following inequality:

$$
\int_{T_{i}}\left(\omega-\gamma_{h} \omega\right)^{2} d \mathbf{x} \leqslant C h^{2}|\omega|_{1, T_{i}}^{2}, \quad i=2,3
$$

This completes the proof of (38) by (48) and (49).

Lemma 5. For any $\omega \in H_{h}$ and $e \in \varepsilon_{h}$, one has

$$
\begin{gathered}
\left\|\left[\gamma_{h} \omega\right]\right\|_{L^{2}(e)} \leqslant\|[\omega]\|_{L^{2}(e)} ; \\
\left\|\left[\omega-\gamma_{h} \omega\right]\right\|_{L^{2}(e)} \leqslant 2\|[\omega]\|_{L^{2}(e)} .
\end{gathered}
$$

Proof. If $e$ is the common side of $T_{1}, T_{2} \in \mathscr{T}_{h}^{*}$, and $T_{1} \subset K_{1}$, $T_{2} \subset K_{2}$, by the definition of $\gamma_{h}$, we have

$$
\begin{aligned}
\left\|\left[\gamma_{h} \omega\right]\right\|_{L^{2}(e)}^{2} & =\left\|\left.\gamma_{h} \omega\right|_{\partial T_{1}} \cdot \mathbf{n}_{1}+\left.\gamma_{h} \omega\right|_{\partial T_{2}} \cdot \mathbf{n}_{2}\right\|_{L^{2}(e)}^{2} \\
& =\left\|h_{e}^{-1}\left(\left.\int_{e} \omega\right|_{T_{1}} d s \cdot \mathbf{n}_{1}+\left.\int_{e} \omega\right|_{T_{2}} d s \cdot \mathbf{n}_{2}\right)\right\|_{L^{2}(e)}^{2} \\
& =h_{e}^{-2}\left\|\int_{e}[\omega] d s\right\|_{L^{2}(e)}^{2} \\
& =h_{e}^{-1}\left(\int_{e}[\omega] d s\right)^{2} .
\end{aligned}
$$

Using the Hölder inequality, we can get

$$
\left\|\left[\gamma_{h} \omega\right]\right\|_{L^{2}(e)}^{2} \leqslant \int_{e}[\omega]^{2} d s=\|[\omega]\|_{L^{2}(e)}^{2} .
$$

If $e \in \partial \Omega$, there exists a $T \in \mathscr{T}_{h}^{*}$, such that $e \in \partial T$ and $T \subset K$; we have

$$
\begin{aligned}
\left\|\left[\gamma_{h} \omega\right]\right\|_{L^{2}(e)}^{2} & =\left\|\gamma_{h} \omega \cdot \mathbf{n}\right\|_{L^{2}(e)}^{2} \\
& =\left\|\left.h_{e}^{-1} \int_{e} \omega\right|_{T} d s \cdot \mathbf{n}\right\|_{L^{2}(e)}^{2} \\
& =h_{e}^{-1}\left(\int_{e}[\omega] d s\right)^{2} \\
& \leqslant\|[\omega]\|_{L^{2}(e)}^{2} .
\end{aligned}
$$

Thus, (50) is valid.

For (51), we have

$$
\begin{aligned}
\left\|\left[\omega-\gamma_{h} \omega\right]\right\|_{L^{2}(e)} & =\left\|[\omega]-\left[\gamma_{h} \omega\right]\right\|_{L^{2}(e)} \\
& \leqslant 2\|[\omega]\|_{L^{2}(e)}
\end{aligned}
$$

following from (50).

Lemma 6 (see [20]). Let $\mathscr{T}$ be a regular triangulation; then, there exists a constant $C>0$ independent of $h_{K}$ such that, for $w \in H^{1}(K)$ and $K \in \mathscr{T}_{h}$, the following inequality holds:

$$
\int_{\partial K}|w|^{2} d s \leqslant C\left\{h_{K}^{-1}\|w\|_{0, K}^{2}+h_{K}|w|_{1, K}^{2}\right\} .
$$

\section{Existence, Uniqueness, and Convergence of DIFVE Solution}

In this section, we will prove the existence and uniqueness of the solution to (24) and conduct its convergence analysis in the broken ||$|\cdot|||$ norm.

Lemma 7. There is a constant $C$ independent of $h$ such that for $\sigma_{0}$ large enough and $\epsilon$ small enough

$$
a_{h}^{*}\left(u_{h}, \gamma_{h} u_{h}\right) \geqslant\left. C\left|\| u_{h}\right|\right|^{2} .
$$


Proof. By Cauchy-Schwarz's inequality, we have

$$
\begin{aligned}
& \sum_{e \in \varepsilon_{h}^{*}} \int_{e}\left[\mathbb{B} \nabla u_{h} \cdot \mathbf{n}\right]\left\{u_{h}-\gamma_{h} u_{h}\right\} d s \\
& \quad \leqslant\left(\sum_{e \in \varepsilon_{h}^{*}}\left\|\left[\mathbb{B} \nabla u_{h} \cdot \mathbf{n}\right]\right\|_{L^{2}(e)}^{2}\right)^{1 / 2}\left(\sum_{e \in \varepsilon_{h}^{*}}\left\|\left\{u_{h}-\gamma_{h} u_{h}\right\}\right\|_{L^{2}(e)}^{2}\right)^{1 / 2} .
\end{aligned}
$$

Using the trace inequality (56), we have

$$
\begin{aligned}
& \left\|\left\{u_{h}-\gamma_{h} u_{h}\right\}\right\|_{L^{2}(e)}^{2} \\
& \quad \leqslant \frac{1}{2}\left(\left\|u_{h}-\left.\gamma_{h} u_{h}\right|_{K_{1}^{e}}\right\|_{L^{2}(e)}^{2}+\left\|u_{h}-\left.\gamma_{h} u_{h}\right|_{K_{2}^{e}}\right\|_{L^{2}(e)}^{2}\right) \\
& \quad \leqslant C\left(h_{K_{1}^{e}}\left|u_{h}\right|_{1, K_{1}}^{2}+h_{K_{2}^{e}}\left|u_{h}\right|_{1, K_{2}}^{2}\right) \\
& \quad \leqslant C h\left(\left|u_{h}\right|_{1, K_{1}}^{2}+\left|u_{h}\right|_{1, K_{2}}^{2}\right),
\end{aligned}
$$

where edge $e$ is shared by the elements $K_{1}^{e}$ and $K_{2}^{e}$. Therefore,

$$
\left(\sum_{e \in \varepsilon_{h}^{*}}\left\|\left\{u_{h}-\gamma_{h} u_{h}\right\}\right\|_{L^{2}(e)}^{2}\right)^{1 / 2} \leqslant C h^{1 / 2}\left(\sum_{K \in \mathscr{T}_{h}^{m}}\left|u_{h}\right|_{1, k}^{2}\right)^{1 / 2} .
$$

By Young's inequality, we have, for $\varepsilon>0$,

$$
\begin{aligned}
& \sum_{e \in \varepsilon_{h}^{*}} \int_{e}\left[\mathbb{B} \nabla u_{h} \cdot \mathbf{n}\right]\left\{u_{h}-\gamma_{h} u_{h}\right\} d s \\
& \quad \leqslant C h^{1 / 2}\left(\sum_{K \in \mathscr{T}_{h}^{m}}\left|u_{h}\right|_{1, k}^{2}\right)^{1 / 2}\left(\sum_{e \in \varepsilon_{h}^{*}}\left\|\left[\mathbb{B} \nabla u_{h} \cdot \mathbf{n}\right]\right\|_{L^{2}(e)}^{2}\right)^{1 / 2} \\
& \quad \leqslant C_{0} \sum_{e \in \varepsilon_{h}^{*}} h\left\|\left[\mathbb{B} \nabla u_{h} \cdot \mathbf{n}\right]\right\|_{L^{2}(e)}^{2}+\frac{\varepsilon}{3} \sum_{K \in T_{h}^{m}}\left|u_{h}\right|_{1, k}^{2} .
\end{aligned}
$$

Similarly, we obtain

$$
\begin{aligned}
\sum_{e \in \varepsilon_{h}^{*}} \int_{e}\left\{\mathbb{B} \nabla u_{h} \cdot \mathbf{n}\right\}\left[u_{h}-\gamma_{h} u_{h}\right] d s \\
\leqslant\left(\sum_{e \in \varepsilon_{h}^{*}}\left\|\left\{\mathbb{B} \nabla u_{h} \cdot \mathbf{n}\right\}\right\|_{L^{2}(e)}^{2}\right)^{1 / 2} \\
\times\left(\sum_{e \in \varepsilon_{h}^{*}}\left\|\left[u_{h}-\gamma_{h} u_{h}\right]\right\|_{L^{2}(e)}^{2}\right)^{1 / 2} .
\end{aligned}
$$

On the one hand, we have

$$
\left\|\left\{\mathbb{B} \nabla u_{h} \cdot \mathbf{n}\right\}\right\|_{L^{2}(e)}^{2} \leqslant C h^{-1}\left(\left|u_{h}\right|_{1, K_{1}}^{2}+\left|u_{h}\right|_{1, K_{2}}^{2}\right),
$$

and thus

$$
\left(\sum_{e \in \varepsilon_{h}^{*}}\left\|\left\{\mathbb{B} \nabla u_{h} \cdot \mathbf{n}\right\}\right\|_{L^{2}(e)}^{2}\right)^{1 / 2} \leqslant C h^{-1 / 2}\left(\sum_{K \in \mathscr{T}_{h}^{m}}\left|u_{h}\right|_{1, k}^{2}\right)^{1 / 2} .
$$

On the other hand, we get

$$
\left(\sum_{e \in \varepsilon_{h}^{*}}\left\|\left[u_{h}-\gamma_{h} u_{h}\right]\right\|_{L^{2}(e)}^{2}\right)^{1 / 2} \leqslant 2\left(\sum_{e \in \varepsilon_{h}^{*}}\left\|\left[u_{h}\right]\right\|_{L^{2}(e)}^{2}\right)^{1 / 2}
$$

following from (51). Therefore,

$$
\begin{aligned}
\sum_{e \in \varepsilon_{h}^{*}} \int_{e}\left\{\mathbb{B} \nabla u_{h} \cdot \mathbf{n}\right\}\left[u_{h}-\gamma_{h} u_{h}\right] d s \\
\leqslant h^{-1 / 2}\left(\sum_{K \in \mathscr{T}_{h}^{m}}\left|u_{h}\right|_{1, K}^{2}\right)^{1 / 2}\left(\sum_{e \in \varepsilon_{h}^{*}}\left\|\left[u_{h}\right]\right\|_{L^{2}(e)}^{2}\right)^{1 / 2} \\
\leqslant C^{\left(\alpha_{0}-1\right) / 2}\left(\sum_{K \in \mathscr{T}_{h}^{m}}\left|u_{h}\right|_{1, K}^{2}\right)^{1 / 2} \\
\quad \times\left(\sum_{e \in \varepsilon_{h}^{*}} \frac{1}{|e|^{\alpha_{0}}}\left\|\left[u_{h}\right]\right\|_{L^{2}(e)}^{2}\right)^{1 / 2}
\end{aligned}
$$

where $\alpha_{0}>1$. By $\varepsilon$-inequality, we have

$$
\begin{aligned}
& \sum_{e \in \varepsilon_{h}^{*}} \int_{e}\left\{\mathbb{B} \nabla u_{h} \cdot \mathbf{n}\right\}\left[u_{h}-\gamma_{h} u_{h}\right] d s \\
& \quad \leqslant \frac{\epsilon}{3} \sum_{K \in \mathscr{T}_{h}^{m}}\left|u_{h}\right|_{1, K}^{2}+C_{1} \sum_{e \in \varepsilon_{h}^{*}} \frac{1}{|e|^{\alpha_{0}}}\left\|\left[u_{h}\right]\right\|_{L^{2}(e)}^{2} .
\end{aligned}
$$

Similarly, we can get

$$
\begin{aligned}
& \sum_{e \in \varepsilon_{h}} \int_{e}\left\{\mathbb{B} \nabla u_{h} \cdot \mathbf{n}\right\}\left[\gamma_{h} u_{h}\right] d s \\
& \quad \leqslant \frac{\epsilon}{3} \sum_{K \in \mathscr{T}_{h}}\left|u_{h}\right|_{1, K}^{2}+C_{2} \sum_{e \in \varepsilon_{h}} \frac{1}{|e|^{\alpha_{0}}}\left\|\left[u_{h}\right]\right\|_{L^{2}(e)}^{2} .
\end{aligned}
$$

Combining (61), (67), and (68), we obtain

$$
\begin{aligned}
& a_{h}^{*}\left(u_{h}, \gamma_{h} u_{h}\right) \\
& \geqslant\left(C-\frac{\epsilon}{3}\right) \sum_{K \in \mathscr{T}_{h}^{n}}\left|u_{h}\right|_{1, K}^{2}+(C-\epsilon) \sum_{K \in \mathscr{T}_{h}^{m}}\left|u_{h}\right|_{1, K}^{2} \\
& \quad+\sum_{e \in \varepsilon_{h}^{*}} \frac{\sigma_{0}-C_{1}-C_{2}}{|e|^{\alpha_{0}}}\left\|\left[u_{h}\right]\right\|_{L^{2}(e)}^{2} \\
& \quad+\sum_{e \in \varepsilon_{h} \backslash \varepsilon_{h}^{*}} \frac{\sigma_{0}-C_{2}}{|e|^{\alpha_{0}}}\left\|\left[u_{h}\right]\right\|_{L^{2}(e)}^{2} \\
& \quad+\sum_{e \in \varepsilon_{h}^{*}} C_{0} h\left\|\left[\mathbb{B} \nabla u_{h} \cdot \mathbf{n}\right]\right\|_{L^{2}(e)}^{2} .
\end{aligned}
$$

Choosing $\sigma_{0}$ large enough and $\epsilon$ small enough, we have

$$
a_{h}^{*}\left(u_{h}, \gamma_{h} u_{h}\right) \geqslant\left. C\left|\| u_{h}\right|\right|^{2}
$$


Lemma 8. For $u, \omega \in H_{h}$, one has

$$
\begin{aligned}
& a_{h}^{*}\left(u, \gamma_{h} \omega\right) \\
& \quad \leqslant C+\left(\||u|\|+\left(\sum_{K \in \mathscr{T}_{h}} h^{2}|u|_{\widetilde{H}^{2}(K)}^{2}\right)^{1 / 2}\right)\||\omega|\| .
\end{aligned}
$$

If $u_{h}, \omega_{h} \in S_{h}(\Omega)$, then

$$
a_{h}^{*}\left(u_{h}, \gamma_{h} \omega_{h}\right) \leqslant C\left\|\left|u_{h}\right|\right\| \mid\left\|\omega_{h}\right\| \| .
$$

Proof. By (38) and the trace inequality (56), we have, for any $e^{s} \in \varepsilon_{h}^{*}, s=+,-$,

$$
\begin{aligned}
& \int_{e^{s}} \mathbb{B} \nabla u \cdot \mathbf{n}\left(\omega-\gamma_{h} \omega\right) d s \\
& \quad \leqslant C\left(h^{-1}|u|_{1, K^{s}}^{2}+h|u|_{2, K^{s}}^{2}\right)^{1 / 2}\left(h^{-1}\left|\omega-\gamma_{h} \omega\right|_{L^{2}\left(K^{s}\right)}^{2}\right. \\
& \left.\quad+h\left|\omega-\gamma_{h} \omega\right|_{1, K^{s}}^{2}\right)^{1 / 2} \\
& \leqslant C\left(|u|_{1, K^{s}}^{2}+h^{2}|u|_{2, K^{s}}^{2}\right)^{1 / 2}|\omega|_{1, K^{s}} \\
& \leqslant C\left(|u|_{1, K}^{2}+h^{2}|u|_{\widetilde{H}^{2}(K)}^{2}\right)^{1 / 2}|\omega|_{1, K} \cdot
\end{aligned}
$$

By the Cauchy-Schwarz inequality, we obtain

$$
\begin{aligned}
& |A(u, \omega)| \\
& \leqslant\left|\sum_{K \in \mathscr{T}_{h}} \int_{K} \mathbb{B} \nabla u \nabla \omega d x\right| \\
& +\left|\sum_{K \in \mathscr{T}_{h}^{m}} \sum_{e \in \varepsilon_{h}^{*}} \int_{e} B \nabla u \cdot \mathbf{n}\left(\omega-\gamma_{h} \omega\right) d s\right| \\
& \leqslant C\left(|u|_{1, h}+\left(\sum_{K \in \mathscr{T}_{h}}\left(|u|_{1, K}^{2}+h^{2}|u|_{\widetilde{H}^{2}(K)}^{2}\right)\right)^{1 / 2}\right)|\omega|_{1, h} .
\end{aligned}
$$

The definition of $a_{h}\left(u, \gamma_{h} \omega\right)$ and the inequality above imply that

$$
\begin{aligned}
\left|a_{h}\left(u, \gamma_{h} \omega\right)\right| \leqslant & C\left\{|u|_{1, h}|\omega|_{1, h}\right\} \\
& +\left(\sum_{K \in \mathscr{T}_{h}}\left(|u|_{1, K}^{2}+h^{2}|u|_{\widetilde{H}^{2}(K)}^{2}\right)\right)^{1 / 2}|\omega|_{1, h}
\end{aligned}
$$

$$
\begin{aligned}
& +\left(\sum_{K \in \mathscr{T}_{h}}\left(|u|_{1, K}^{2}+h^{2}|u|_{\widetilde{H}^{2}(K)}^{2}\right)\right)^{1 / 2} \\
& \times\left(\sum_{e \in \varepsilon_{h}}\|[\omega]\|_{L^{2}(e)}^{2}\right)^{1 / 2} \\
& +\left(\sum_{e \in \varepsilon_{h}}\|[u]\|_{L^{2}(e)}^{2}\right)^{1 / 2}\left(\sum_{e \in \varepsilon_{h}}\|[\omega]\|_{L^{2}(e)}^{2}\right)^{1 / 2} \\
& \leqslant C\left(\|\| u \| \mid+\left(\sum_{K \in \mathscr{T}_{h}} h^{2}|u|_{\widetilde{H}^{2}(K)}^{2}\right)^{1 / 2}\right) \mid\|\omega\| \| .
\end{aligned}
$$

This completes the proof of (71). For (72), we can get the following from (71) and $\left|u_{h}\right|_{\widetilde{H}^{2}(K)}=0, \forall u_{h} \in S_{h}(\Omega)$.

Lemmas 7 and 8 guarantee the existence and uniqueness of the discontinuous immersed finite volume element solution to (23) when choosing $\sigma_{0}$ large enough.

\section{Error Estimates in the Energy Norm}

We will derive an optimal-order error estimate in the norm |II - II defined in (35) and a first order error estimate in $L^{2}$ norm. We start with the following lemmas.

Lemma 9. Let $u$ be the solutions of (1); one has the conclusion

$$
\left\|u-\Pi_{h} u\right\| \mid \leqslant C h\|u\|_{\widetilde{H}^{2}(\Omega)} .
$$

Proof. By the definition of norm ||$|\cdot|||$, we have

$$
\begin{aligned}
\| u- & \Pi_{h} u||^{2} \\
= & \left|u-\Pi_{h} u\right|_{1, h}^{2}+\sum_{e \in \varepsilon_{h}} \frac{1}{|e|^{\alpha_{0}}}\left\|\left[u-\Pi_{h} u\right]\right\|_{L^{2}(e)}^{2} \\
& +\sum_{e \in \varepsilon_{h}^{*}} h\left\|\left[\nabla\left(u-\Pi_{h} u\right) \cdot \mathbf{n}\right]\right\|_{L^{2}(e)}^{2} .
\end{aligned}
$$

Using trace inequality and (12), we have

$$
\begin{gathered}
\left\|\left[u-\Pi_{h} u\right]\right\|_{L^{2}(e)}^{2} \\
\leqslant C\left(\left\|u-\left.\Pi_{h} u\right|_{K_{1}^{e}}\right\|_{L^{2}(e)}^{2}+\left\|u-\left.\Pi_{h} u\right|_{K_{2}^{e}}\right\|_{L^{2}(e)}^{2}\right) \\
\leqslant C\left(h^{-1}\left\|u-\Pi_{h} u\right\|_{L^{2}\left(K_{1}\right)}^{2}+h\left|u-\Pi_{h} u\right|_{1, K_{1}}^{2}\right. \\
\left.\quad+h^{-1}\left\|u-\Pi_{h} u\right\|_{L^{2}\left(K_{2}\right)}^{2}+h\left|u-\Pi_{h} u\right|_{1, K_{2}}^{2}\right) \\
\leqslant C h^{2}\left(\|u\|_{\widetilde{H}^{2}\left(K_{1}\right)}^{2}+\|u\|_{\widetilde{H}^{2}\left(K_{2}\right)}^{2}\right),
\end{gathered}
$$

where $e$ is shared by the elements $K_{1}$ and $K_{2}$. Thus, we obtain

$$
\sum_{e \in \varepsilon_{h}}\left\|\left[u-\Pi_{h} u\right]\right\|_{L^{2}(e)}^{2} \leqslant C h^{2}\|u\|_{\widetilde{H}^{2}(\Omega)}^{2} .
$$


Analogously, we can get

$$
\sum_{e \in \varepsilon_{h}^{*}} h\left\|\left[\nabla\left(u-\Pi_{h} u\right) \cdot \mathbf{n}\right]\right\|_{L^{2}(e)}^{2} \leqslant C h^{2}\|u\|_{\widetilde{H}^{2}(\Omega)}^{2} .
$$

By (12), (79), and (80), we obtain (76).

Lemma 10 (see [21]). There exists a constant $C$ independent of $h$ such that

$$
\|\omega\| \leqslant C\|\mid \omega\| \|, \quad \forall \omega \in S_{h}(\Omega) .
$$

Theorem 11. Let $u_{h} \in S_{0 h}(\Omega)$ and $u \in \widetilde{H}^{2}(\Omega) \cap H_{0}^{1}(\Omega)$ be the solutions of (23) and (1), respectively; then, there exists a constant $C$ independent of $h$ such that

$$
\begin{gathered}
\left\|u-u_{h}\right\| \mid \leqslant C h\|u\|_{\widetilde{H}^{2}(\Omega)}, \\
\left\|u-u_{h}\right\| \leqslant C h\|u\|_{\widetilde{H}^{2}(\Omega)} .
\end{gathered}
$$

Proof. Subtracting (25) from (23) gives

$$
a_{h}^{*}\left(u-u_{h}, \gamma_{h} \omega_{h}\right)=0, \quad \forall \omega_{h} \in S_{0 h}(\Omega) .
$$

Using (71), (76), and (84), we have

$$
\begin{aligned}
&\|\| u_{h}-\Pi_{h} u\|\|^{2} \\
& \leqslant C a_{h}^{*}\left(u_{h}-\Pi_{h} u_{h}, \gamma_{h}\left(u_{h}-\Pi_{h} u_{h}\right)\right) \\
&= C a_{h}^{*}\left(u-\Pi_{h} u, \gamma_{h}\left(u_{h}-\Pi_{h} u_{h}\right)\right) \\
& \leqslant C\left(\left\|u-\Pi_{h} u\right\| \|+\left(\sum_{K \in \mathscr{T}_{h}} h^{2}\left|u-\Pi_{h} u\right|_{\widetilde{H}^{2}(K)}^{2}\right)^{1 / 2}\right) \\
& \times\left\|u_{h}-\Pi_{h} u\right\| \\
& \leqslant C h^{2}\|u\|_{\widetilde{H}^{2}(\Omega)}^{2} .
\end{aligned}
$$

Thus, we can get

$$
\left\|u-u_{h} \mid\right\| \leqslant C h\|u\|_{\widetilde{H}^{2}(\Omega)}
$$

following from triangle inequality, (76), and (85).

For (83), we can get by (12), (81) and triangle inequality. We have completed the proof.

\section{Conflict of Interests}

The authors declare that there is no conflict of interests regarding the publication of this paper.

\section{Acknowledgments}

This work is supported by the NSF of China under Grants 10971254 and 10926100 and the Development projects of Shandong Province Science and Technology 2012GGB02298.

\section{References}

[1] J. H. Bramble, "A finite element method for interface problems in domains with smooth boundaries and interfaces," Advances in Computational Mathematics, vol. 6, no. 1, pp. 109-138, 1996.

[2] I. Babuska, "The finite element method for elliptic equations with discontinuous coefficients," Computing, vol. 5, no. 3, pp. 207-213, 1970

[3] Z. Chen and J. Zou, "Finite element methods and their convergence for elliptic and parabolic interface problems," Numerische Mathematik, vol. 79, no. 2, pp. 175-202, 1998.

[4] R. J. Leveque and Z. Li, “The immersed interface method for elliptic equations with discontinuous coefficients and singular sources," SIAM Journal on Numerical Analysis, vol. 31, no. 4, pp. 1019-1044, 1994.

[5] Z. Li, T. Lin, and X. Wu, "New Cartesian grid methods for interface problems using the finite element formulation," Numerische Mathematik, vol. 96, no. 1, pp. 61-98, 2003.

[6] Y. Gong, B. Li, and Z. Li, "Immersed-interface finite-element methods for elliptic interface problems with nonhomogeneous jump conditions," SIAM Journal on Numerical Analysis, vol. 46, no. 1, pp. 472-495, 2008.

[7] Z. Li, T. Lin, Y. Lin, and R. C. Rogers, "An immersed finite element space and its approximation capability," Numerical Methods for Partial Differential Equations, vol. 20, no. 3, pp. 338367, 2004.

[8] Z. Li, "The immersed interface method using a finite element formulation," Applied Numerical Mathematics, vol. 27, no. 3, pp. 253-267, 1998.

[9] Z. Li and K. Ito, The Immersed Interface Method: Numerical Solutions of PDEs Involving Interfaces and Irregular Domains, vol. 33 of Frontiers in Applied Mathematics, SIAM, Philadelphia, Pa, USA, 2006.

[10] R. E. Ewing, Z. Li, T. Lin, and Y. Lin, "The immersed finite volume element methods for the elliptic interface problems," Mathematics and Computers in Simulation, vol. 50, no. 1-4, pp. 63-76, 1999.

[11] Z. Cai, "On the finite volume element method," Numerische Mathematik, vol. 58, no. 1, pp. 713-735, 1991.

[12] S. H. Chou, "Analysis and convergence of a covolume method for the generalized stokes problem," Mathematics of Computation, vol. 66, no. 217, pp. 85-104, 1997.

[13] Z. Cai and S. Mccormick, "On the accuracy of the finite volume element method for diffusion equations on composite grids," SIAM Journal on Numerical Analysis, vol. 27, no. 3, pp. 636-655, 1990.

[14] R. E. Ewing, R. D. Lazarov, and Y. Lin, "Finite volune element approximations of non-local in time one-dimensional reactive flows in porous media," Tech. Rep. ISC-98-06-MATH, Institute for Scientific Computation.

[15] E. Süli, "Convergence of finite volume schemes for Poisson's equation on nonuniform meshes," SIAM Journal on Numerical Analysis, vol. 28, no. 5, pp. 1419-1430, 1991.

[16] J. M. Thomas and D. Trujillo, "Analysis of finite volume methods," Tech. Rep. 19, Université de Pau et des Pays de L'adour, Pau, France, 1995.

[17] N. An and H. Z. Chen, "A partially penaltyimmersed finite element method for anisotropic flow medels in porous medium," Numerical Methods for PDE. In press.

[18] R. G. Zhang and H. Z. Chen, "An immersed finite element method for anisotropic flow models in porous medium," in 
Proceedings of the International Conference on Information Science and Technology (ICIST '11), pp. 168-175, Nanjing, China, March 2011.

[19] D. N. Arnold, F. Brezzi, B. Cockburn, and L. Donatella Marini, "Unified analysis of discontinuous Galerkin methods for elliptic problems," SIAM Journal on Numerical Analysis, vol. 39, no. 5, pp. 1749-1779, 2002.

[20] B. Rivière, Discontinuous Galerkin Methods for Solving Elliptic and Parabolic Equations: Theory and Implementation, vol. 35 of Frontiers in Applied Mathematics, SIAM, Philadelphia, Pa, USA, 2008.

[21] X. Ye, "A new discontinuous finite volume method for elliptic problems," SIAM Journal on Numerical Analysis, vol. 42, no. 3, pp. 1062-1072, 2004. 


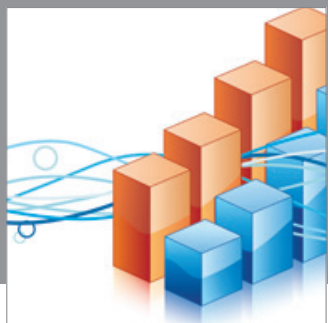

Advances in

Operations Research

mansans

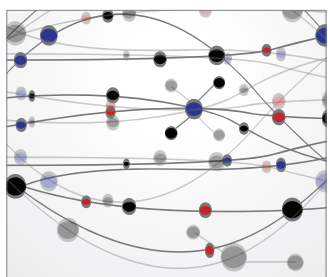

The Scientific World Journal
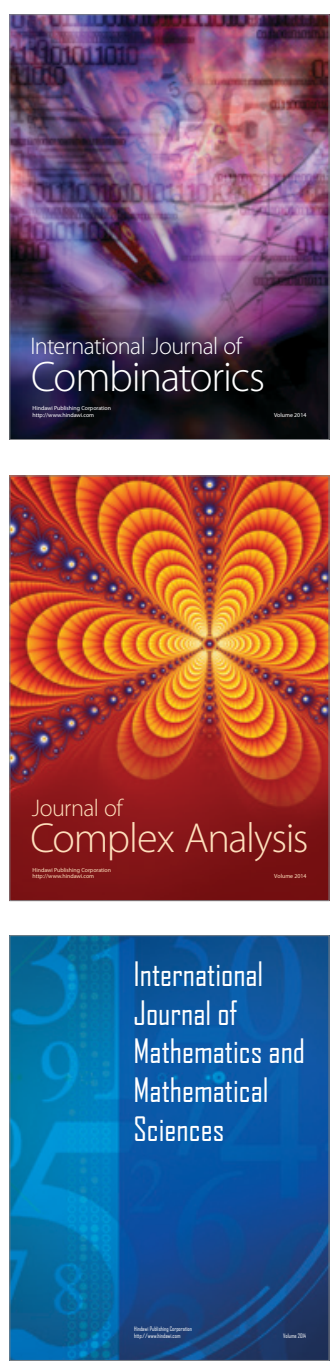
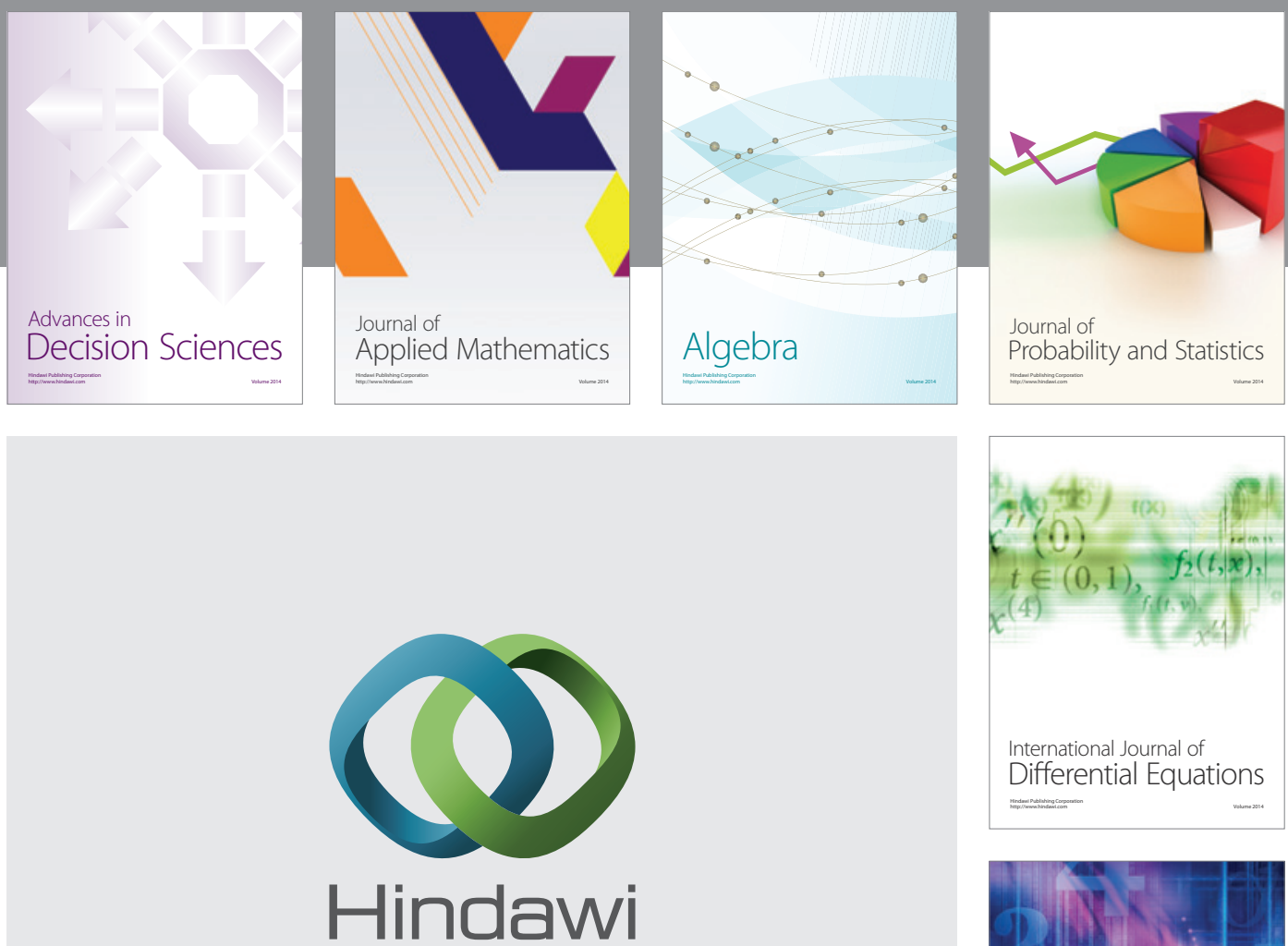

Submit your manuscripts at http://www.hindawi.com
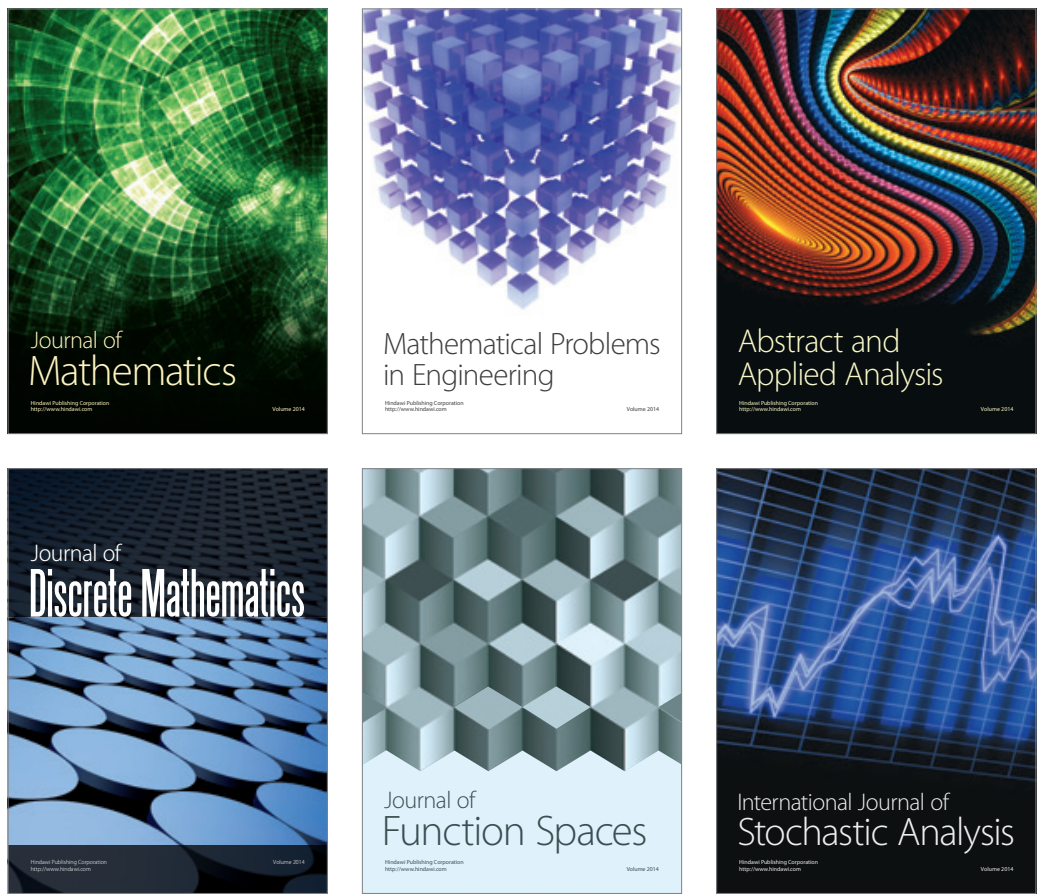

Journal of

Function Spaces

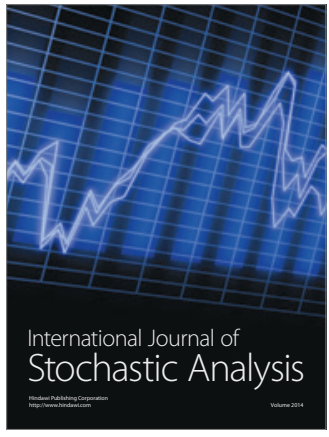

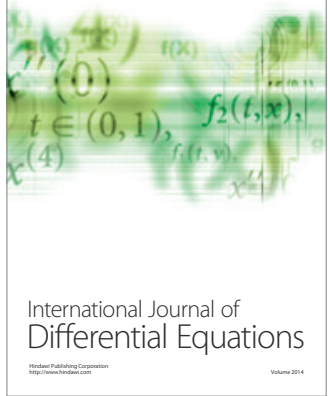
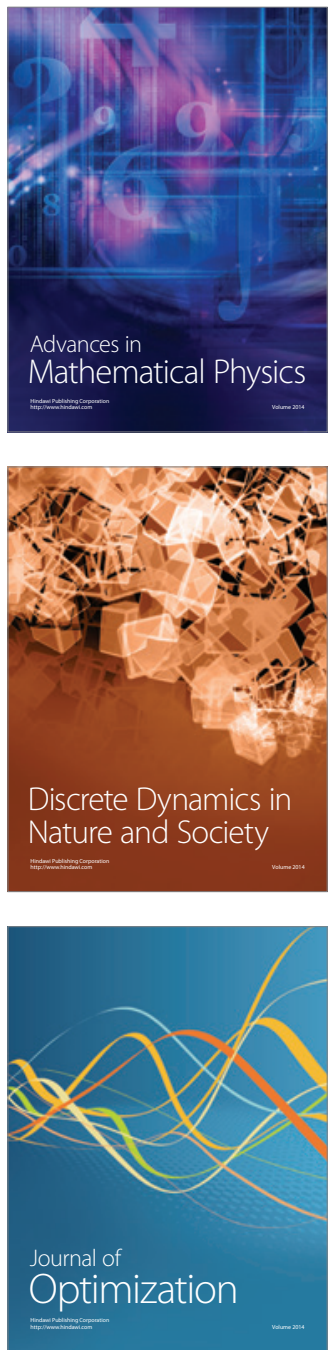